\title{
Temporäre Tracheostomie beim Kleintier
}

\author{
Manuel Schmidli, Vetsuisse Fakultät Universität Bern, Schweiz
}

\section{Indikationen}

Die temporäre Tracheostomie mit dem Einsatz eines Tracheostomietubus ist bei verschiedensten, hauptsächlich akuten Erkrankungen der oberen Atemwege mit schwerer Atemnot indiziert.

Beispiele dafür sind:

- Trauma

- Anaphylaxie

- Neoplasie

- morphologische Defekte

Außerdem hat sich diese Technik bei ausgewählten Operationen in der Maulhöhle als nützlich erwiesen, da so mehr Platz und somit bessere Übersicht gewährleistet ist.

\section{Benötigtes Material}

Da temporäre Tracheostomien meist als Notfall bei schwer dyspnoeischen Patienten mit oberer Atemwegsobstruktion durchgeführt werden, muss das benötigte Material unter Umständen schnell zur Verfügung stehen. Es sollte deshalb stets an einem geeigneten Ort bzw. an jenem Ort, an dem normalerweise Notfälle stabilisiert werden, bereitliegen.

Zu den benötigten Materialien zählen:

- Tracheostomietubi in verschiedenen Größen (ca. 2-8 mm Durchmesser; gecufft oder ungecufft)

- steriles OP-Besteck

- genügend starkes Nahtmaterial

- Gaze für die Fixierung des Tubus um den Hals

Für ein adäquates Tubusmanagement werden sterile Kochsalzlösung, Chlorhexidin (2\%) sowie sterile Saugkatheter mit dem dazu passenden Saugsystem benötigt. Zum Schutz des Tracheostomietubus sollten ausreichend Verbandsmaterial und Wundsalbe vorhanden sein.

\section{Management}

Aufgrund des Fremdkörpers in Form des Tubus wird natürlicherweise mehr Sekret in der Trachea gebildet. Dies führt zum erhöhten Risiko einer Obstruktion durch Mukus im Lumen des Tubus. Da dies verheerende Konsequenzen haben kann, muss der Tubus regelmäßig auf mögliche Obstruktionen überprüft und jeweils gereinigt werden.

Falls der Tracheostomietubus einen Cuff hat, sollte dieser nur im Falle einer Beatmung benutzt werden, wenn also der Druck in der Lunge ansonsten nicht aufgebaut werden kann (positive pressure ventilation) oder wenn durch den Tubus Inhalationsanästhetika verabreicht werden sollten. Um den Schaden an der Mukosa der Trachea möglichst minimal zu halten, sollte der Cuff nur ganz vorsichtig aufgeblasen werden. Zudem sollte dieser ungefähr alle 4 Stunden gelöst, der Tubus neu platziert und danach wieder aufgeblasen werden. So werden Drucknekrosen in der Wand der Trachea möglichst gering gehalten.

\section{Online zu finden unter}

https://doi.org/10.1055/s-0043-118892

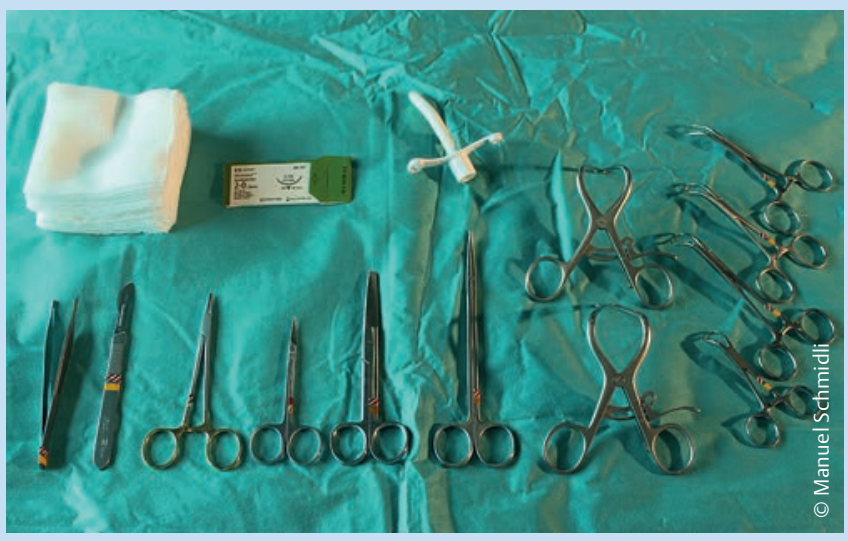

Abb. 1 Benötigte Materialien für die temporäre Tracheostomie.

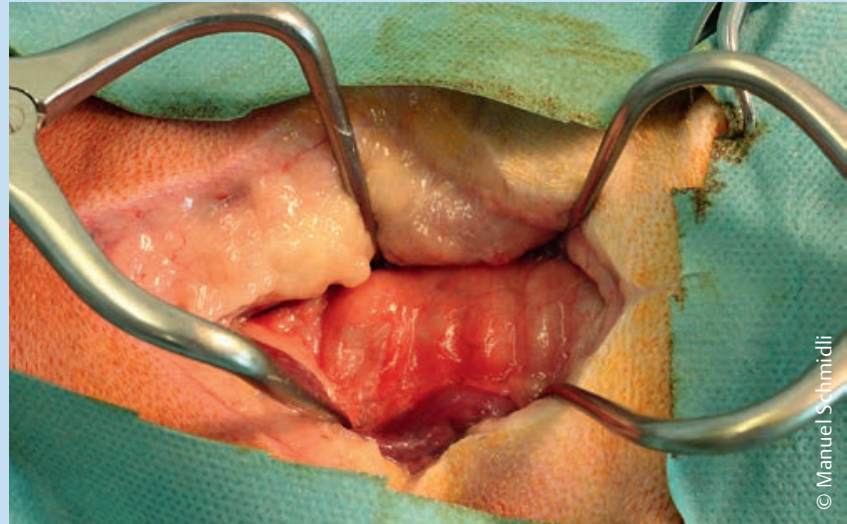

Abb. 2 Freilegung der Trachea: Die Inzision wird in der ventralen zervikalen Mittellinie ausgehend vom Cartilagus cricoideus etwa $3 \mathrm{~cm}$ nach kaudal, zuerst durch die Haut und danach durch das subkutane Gewebe geführt. Anschließend wird der M. sternohyoideus ebenfalls in der Mittellinie in der Aponeurose separiert, um die Trachea freilegen zu können. 


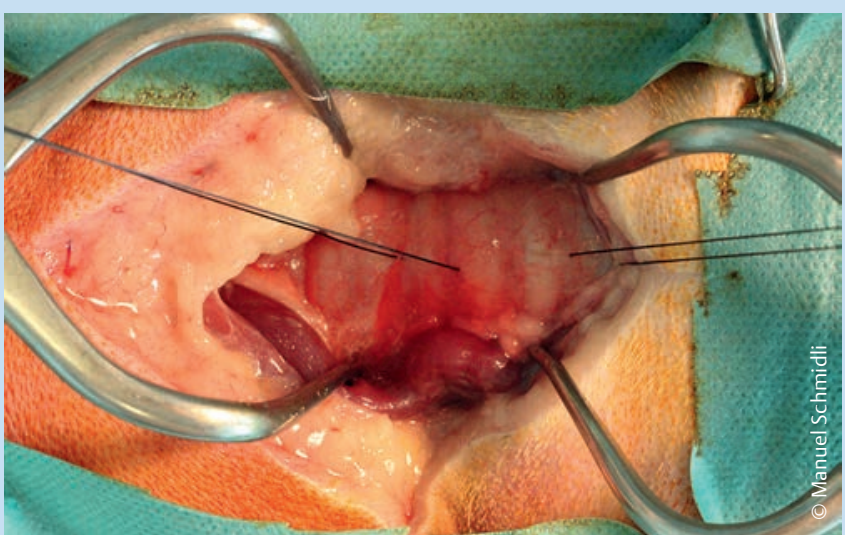

Abb. 3 Setzen der Haltefäden: Abhängig von der Richtung des Schnittes müssen nun Haltefäden gesetzt werden. Bei einer horizontalen Inzision sollten diese um die anliegenden Trachealringe und bei einer vertikalen Inzision jeweils durch die anliegenden Trachealringe gezogen werden. Die Haltefäden ermöglichen, das Lumen der Trachea jederzeit aufrechtzuerhalten. Die Fäden sollten klar markiert werden, um ein späteres Auswechseln des Tubus zu erleichtern. Die Höhe der Inzision der Trachea wird in der Regel beim 4. - 5. Trachealring gewählt.

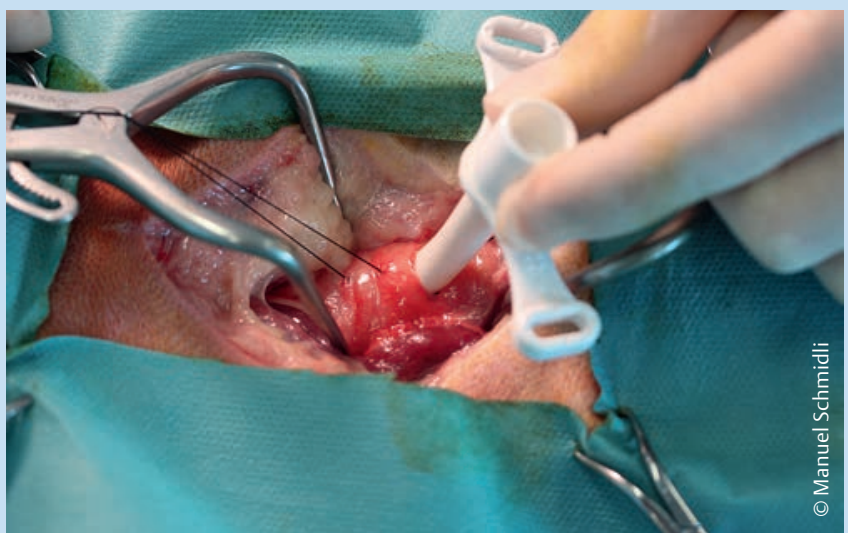

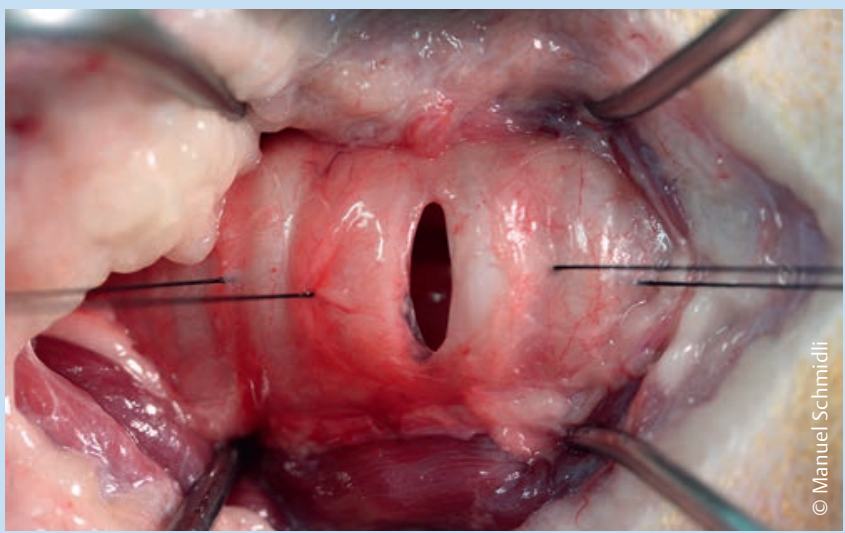

Abb. 4 Tracheostomie: Die Inzision der Trachea kann auf verschiedene Arten erfolgen; entweder horizontal (transversal) zwischen 2 Trachealringen oder alternativ vertikal (longitudinal) über die Länge von 2-3 Trachealringen. Falls ein horizontaler Zugang gewählt wird, sollte die Länge des Schnittes nicht mehr als die Hälfte des Tracheaumfangs betragen.

Abb. 5 Einsetzen des Tracheostomietubus: Ein entsprechender Tubus wird nun vorsichtig durch die vorbereitete Inzision in die Trachea eingesetzt. Sollte viel Blut und Mukus vorhanden sein, muss vorher abgesaugt werden. Die Wahl der Tubusgröße erfolgt je nach Dimension der Trachea. Im Idealfall sollte er eine Größe aufweisen, die genügend Luftfluss gewährleistet, jedoch auch Luftfluss um den Tubus ermöglicht. In der Regel entspricht dies ungefähr der Hälfte des Durchmessers der Trachea. Dies ist insbesondere wichtig, um eine gewisse Absicherung gegen „silent death“ durch eine Okklusion des Tubus zu erreichen.

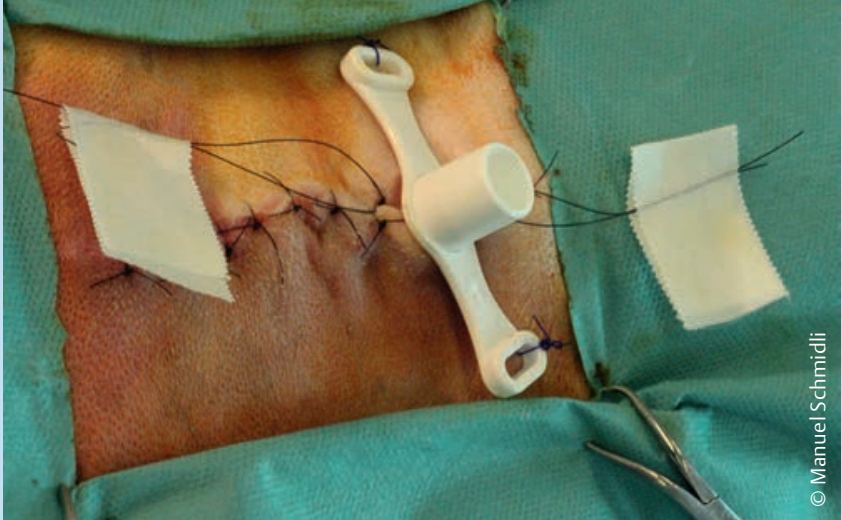

Abb. 6 Fixierung: Kranial und kaudal des Tubus werden M. sternohyoideus, subkutanes Gewebe und Haut routinemäßig adaptiert. Der Tracheostomietubus muss nach dem Einsetzen adäquat mittels entsprechenden Hautknoten oder einer Gaze um den Hals gesichert werden.

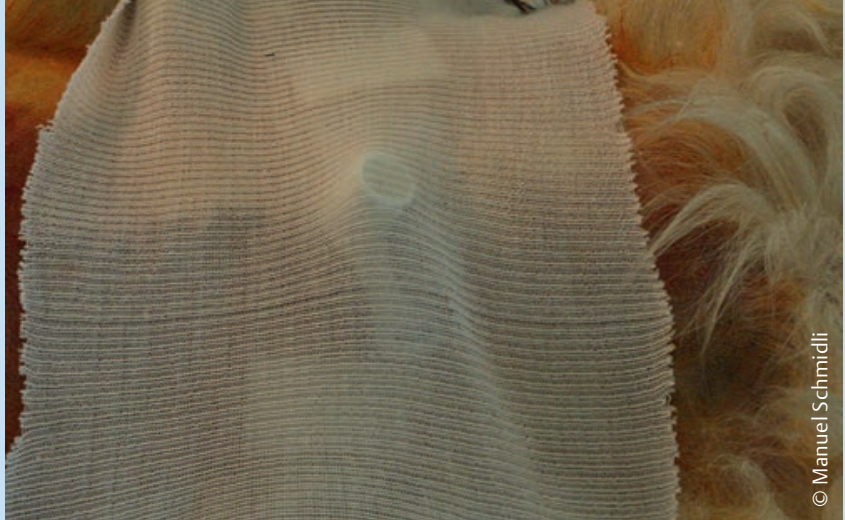

Abb. 7 Schutz: Das Stoma um den Tubus sollte mit einer dünnen Schicht antibiotikahaltiger Salbe abgedeckt oder alternativ mehrmals täglich mit einer $2 \%$ igen Chlorhexidin-Lösung gereinigt werden. Zudem sollte das Stoma leicht bandagiert werden; dünne Strümpfe eignen sich dafür vorzüglich. 